\title{
Disk-halo interactions: molecular clouds in the Galactic center
}

\author{
D. Riquelme ${ }^{1}$, J. Martín-Pintado ${ }^{2}$, R. Mauersberger ${ }^{3}$, S. Martín ${ }^{4}$ \\ and L. Bronfman ${ }^{5}$ \\ ${ }^{1}$ Max-Planck Institute for Radioastronomy, Auf dem Hügel 69, D-53121, Bonn, Germany \\ email: riquelme@mpifr-bonn.mpg.de \\ ${ }^{2}$ Centro de Astrobiología (CSIC/INTA), Ctra. de Torrejón a Ajalvir km 4, E-28850, Torrejón \\ de Ardoz, Madrid, Spain \\ ${ }^{3}$ Joint ALMA Observatory, Alonso de Córdova 3107, Vitacura, Santiago, Chile \\ ${ }^{4}$ IRAM, 300 rue de la Piscine, 38406 Saint Martin dHres, France \\ ${ }^{5}$ Departamento de Astronomía, Universidad de Chile, Casilla 36-D, Santiago, Chile
}

\begin{abstract}
We study the disk-halo interaction, in the context of orbits and Giant Molecular loops (GMLs) in the Galactic center (GC) region. From a large scale survey of the central kpc of the Galaxy, in $\mathrm{SiO} J=(2-1), \mathrm{HCO}^{+} J=(1-0)$ and $\mathrm{H}^{13} \mathrm{CO}^{+} J=(1-0)$ molecular emission, we identify shock regions traced by the enhancement of the $\mathrm{SiO}$. These positions were studied using the ${ }^{12} \mathrm{C} /{ }^{13} \mathrm{C}$ isotopic ratio to trace gas accretion/ejection. We found a systematically higher ${ }^{12} \mathrm{C} /{ }^{13} \mathrm{C}$ isotopic ratio (> 40) toward the GMLs and the $x_{1}$ orbits than for the GC standard molecular clouds $(20-25)$. The high isotopic ratios are consistent with the accretion of the gas from the halo and from the outskirts of the Galactic disk. From multi-transitional observations of $\mathrm{NH}_{3}$, we derive two kinetic temperature regimes (one warm at $\sim 150 \mathrm{~K}$ and one cold at $\sim 40 \mathrm{~K}$ ) for all the positions, except for the GMLs positions where only the warm component is present. The fractional abundances derived from the different molecules support the shock origin for the heating mechanism in the GC. We also present a detailed study of one molecular cloud placed in the foot points of two giant molecular loops, where two of the previously selected positions are placed. Using the $22 \mathrm{~m}$ Mopra telescope we mapped the molecular cloud $\mathrm{M}-3.8+0.9$ in 3 -mm molecular lines. The data show structures at small scale in $\mathrm{SiO}$ emission, with narrower line profiles than those of, e.g, $\mathrm{HCO}^{+}$or $\mathrm{HCN}$, which indicate that the shocks are dynamically confined. The data also show clear differences between different molecular tracers, e.g., between the $\mathrm{SiO}$ and $\mathrm{HCO}^{+}$emission, which would indicate differences in the physical properties and chemistry within the cloud.
\end{abstract}

Keywords. Galaxy: center — ISM: molecules

\section{Introduction}

The Milky Way, and in particular the Galactic center (GC), offers an excellent opportunity to explore in detail the chemical and physical processes associated to the accretion and ejection phenomena present in galactic nuclei. In this work, we study two phenomena which are presumably occurring in the GC region, and can be partly responsible for the gas accretion toward the nuclear region of the Galaxy: the barred potential, and the Giant Molecular Loops (GMLs). Binney et al. (1991) showed that the large-scale gas kinematics in the central regions of the Galaxy can be explained by a barred galactic potential with two major families of orbits inside the bar: the $x_{1}$ orbits parallel to the bar, and the inner $x_{2}$ orbits, orthogonal to it. The gas falls from $x_{1}$ to $x_{2}$ orbits when it self-intersects in the $x_{1}-x_{2}$ orbit transition. The other phenomenon is the huge loop structure (GMLs) found by Fukui et al. (2006) and thought to be formed by a Parker 


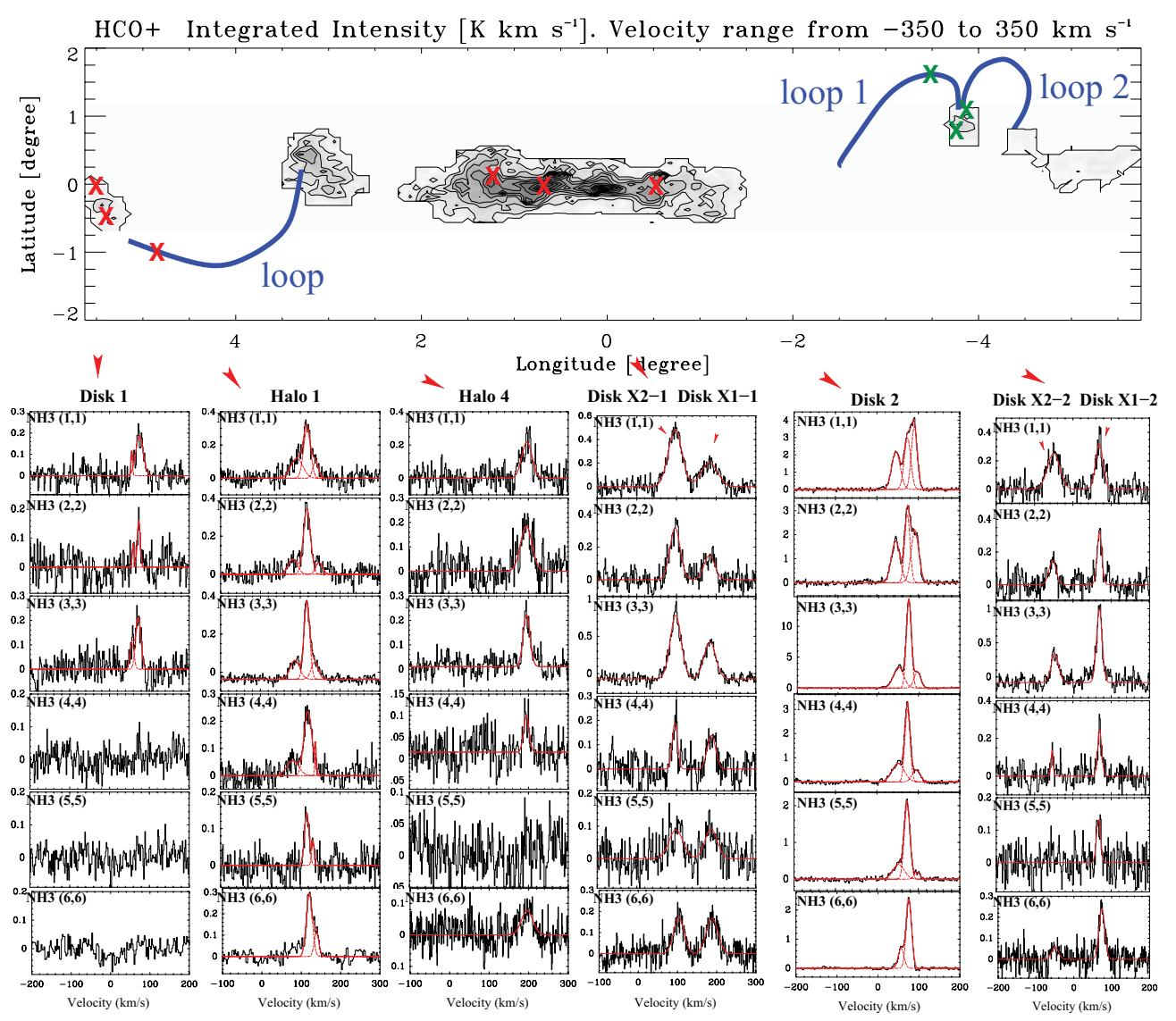

Figure 1. Spectra toward selected positions in the GC in the metastable inversion transitions from $(1,1)$ to $(6,6)$ of $\mathrm{NH}_{3}$ (Riquelme et al. 2013). The positions are indicated in the $\mathrm{HCO}^{+}$ integrated intensity map from Riquelme et al. (2010b). The GMLs found by Fukui et al. (2006) are shown in the figure. [A COLOR VERSION IS AVAILABLE ONLINE.]

instability. The gas in the loops is thought to flow down along the magnetic field lines and join the Galactic plane, generating shock fronts at the foot points of the loops.

\section{Selection of the positions}

We performed a large-scale study of the GC region in $\mathrm{SiO} J=(2-1), \mathrm{HCO}^{+} J=(1-$ 0 ), and $\mathrm{H}^{13} \mathrm{CO}^{+} J=(1-0)$ using the $4 \mathrm{~m}-\mathrm{NANTEN}$ telescope (Riquelme et al. $2010 \mathrm{~b}$ ). We found an increased emission in $\mathrm{SiO}$ as compared to the $\mathrm{HCO}^{+}$line intensity at the foot points of the GMLs and toward the 1.3 complex, which indicates the presence of shocks (Martin-Pintado et al. 1992, Martin-Pintado et al. 1997). From this study we selected 9 positions (see Figure 1) where the $\mathrm{SiO}$ emission shows an enhancement with respect to the $\mathrm{HCO}^{+}$emission. The positions are considered in this work as the "interaction regions", because they mark the places where gas in the GC could be interacting with gas coming from higher latitude (disk-halo interaction) or from larger galactocentric radii, according to the GMLs scenario and the bar potential model, respectively. They were selected including five positions in the GMLs and two positions in the $x_{1}-x_{2}$ orbits interaction places. The positions in the GMLs were called "halo" to differentiate from the molecular clouds in the Galactic plane. Among them, 3 positions are placed where 

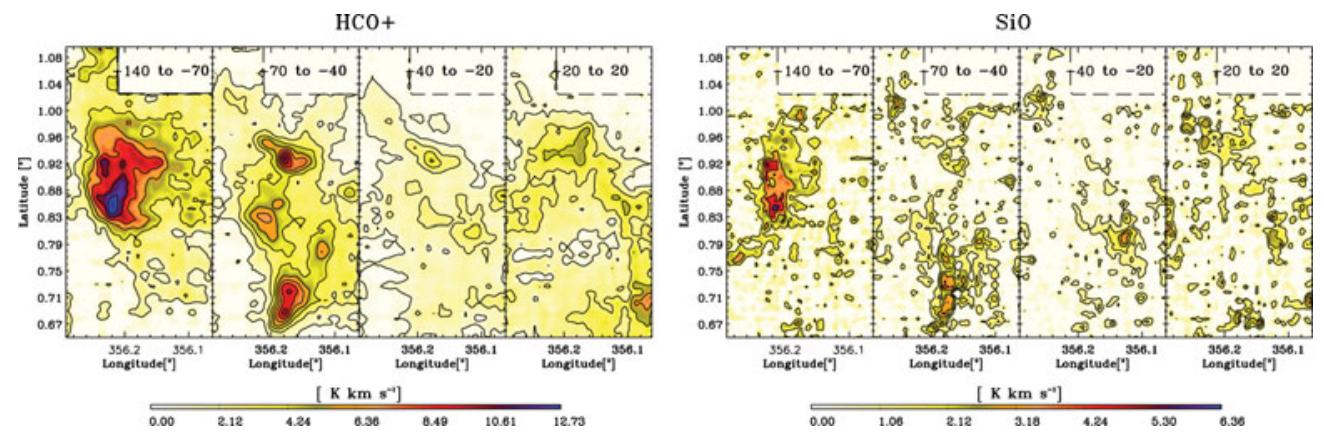

Figure 2. Integrated velocity map in the four selected velocity range (from -110 to $-70 \mathrm{~km} \mathrm{~s}^{-1}$; -70 to $-40 \mathrm{~km} \mathrm{~s}^{-1},-40$ to $-20 \mathrm{~km} \mathrm{~s}^{-1}$ and -20 to $20 \mathrm{~km} \mathrm{~s}^{-1}$ ) in $\mathrm{HCO}^{+}$(left) and $\mathrm{SiO}$ (right). [A COLOR VERSION is AVAILABLE ONLINE.]

the gas in the GMLs joins the gas in the disk (foot points of the loops), and 2 positions are placed at the top of the loops. Each of the positions located at the $x_{1}-x_{2}$ orbits interaction places has two main kinematical components, one associated to the $x_{1}$ orbits and the other to the $x_{2}$ orbits. They were called as "disk X1", and "disk X2". Finally, we selected 2 positions close to $b=0$ (that we called "disk"), which are not associated either with the GMLs nor the location of the orbit interaction, and therefore they should trace the typical GC clouds.

\section{Tracing gas accretion using isotopic ratios}

Using the IRAM 30m-telescope, we observed the $J=(1-0)$ rotational transitions of $\mathrm{HCO}^{+}, \mathrm{HCN}, \mathrm{HNC}$, and their ${ }^{13} \mathrm{C}$ isotopic substitutions toward the 9 selected positions. While ${ }^{12} \mathrm{C}$ is predicted to be formed in first-generation, massive stars on rapid timescales, ${ }^{13} \mathrm{C}$ is produced primarily via $\mathrm{CNO}$ processing of ${ }^{12} \mathrm{C}$ seeds from earlier stellar generations, on a longer timescale. The ${ }^{12} \mathrm{C} /{ }^{13} \mathrm{C}$ isotopic ratio shows, therefore, the relative degree of primary to secondary processing in stars. We found a systematically higher ${ }^{12} \mathrm{C} /{ }^{13} \mathrm{C}$ isotopic ratio $(>40)$ toward the halo and the $x_{1}$ orbits than for the GC standard molecular clouds $(20-25)$. The results are consistent with a scenario where gas from the halo is accreted to the disk and with the transfer of gas from the outskirt of the disk to the GC through $x_{1}$ and $x_{2}$ orbits as suggested by the potential bar scenario (Riquelme et 2010a).

\section{Physical properties of the interaction regions}

We derived the physical conditions of the molecular gas in the interaction regions of the GC. Using the Effelsberg 100m-telescope, we measured the metastable inversion transitions of $\mathrm{NH}_{3}$ from $(1,1)$ to $(6,6)$ in 6 positions that can be observed from this site (Figure 1). Using rotational diagrams and large velocity gradient calculations, we estimated, for the first time, the kinetic temperatures of the molecular clouds in these regions. The kinetic temperatures are high, not only in the typical GC clouds, but also in the high latitude and high velocity clouds observed in this work. Therefore, the heating mechanism of the molecular clouds are likely to be a general characteristics of the molecular gas throughout the GC region. We derive two kinetic temperature regimes (one warm at $\sim 150 \mathrm{~K}$ and one cold at $\sim 40 \mathrm{~K}$ ) for all the positions, except for the halo where only the warm component is present. 


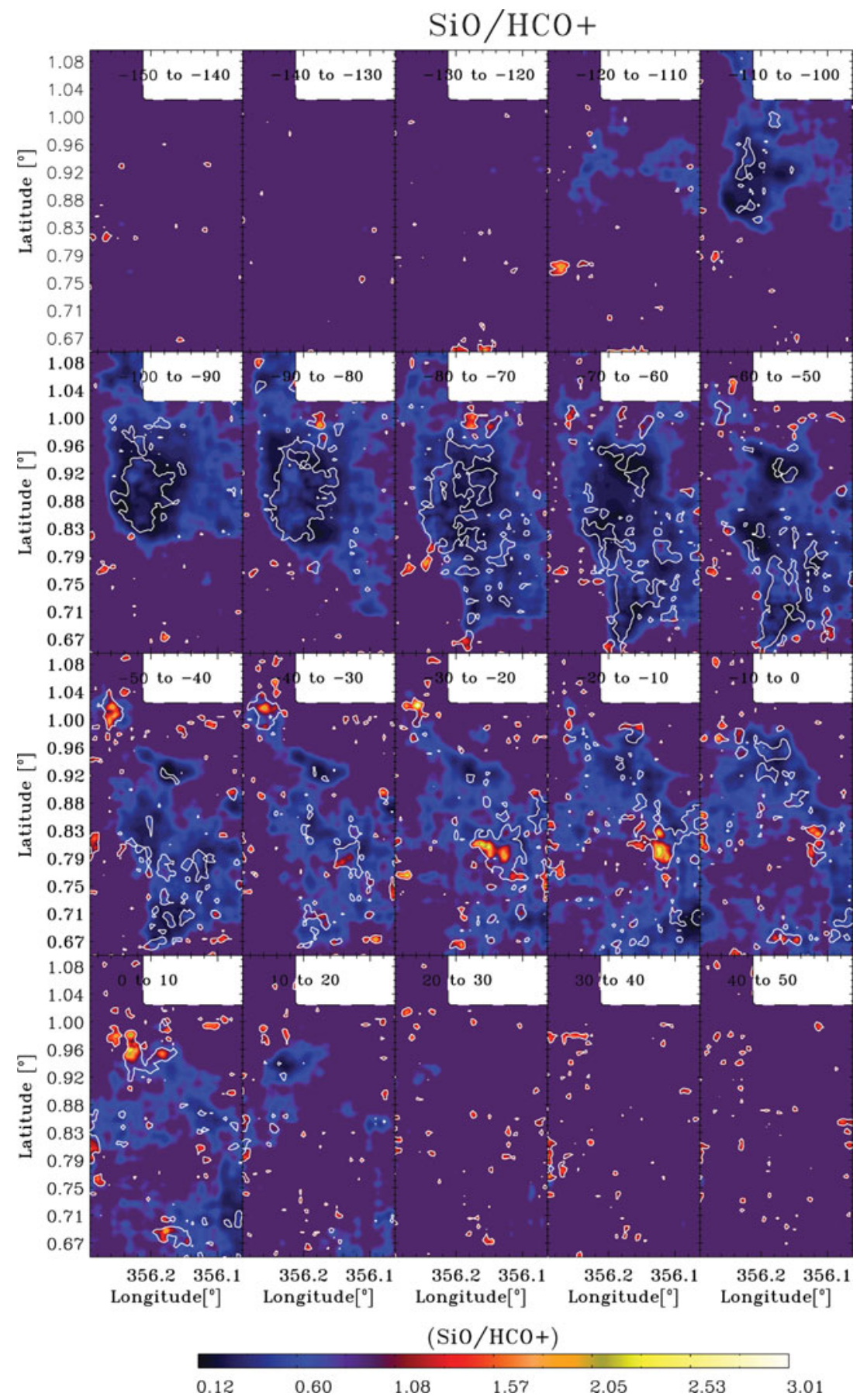

Figure 3. Ratio of the integrated intensity of $\mathrm{SiO} J=(2-1)$ to $\mathrm{HCO}^{+}$, in velocity intervals of $10 \mathrm{~km} \mathrm{~s}^{-1}$. [A COLOR VERSION IS AVAILABLE ONLINE.]

\section{A complete study of the foot point of the GMLs}

We used the Mopra telescope to map one molecular cloud $(\mathrm{M}-3.8+0.9)$ at the foot points of the GMLs in $3 \mathrm{~mm}$ lines. We identified four main velocity components: from -140 to $-70 \mathrm{~km} \mathrm{~s}^{-1}$, from -70 to $-40 \mathrm{~km} \mathrm{~s}^{-1}$, from -40 to $-20 \mathrm{~km} \mathrm{~s}^{-1}$, and from -20 
to $20 \mathrm{~km} \mathrm{~s}^{-1}$. The maps reveal structures at small scales in the $\mathrm{SiO}$ emission, and large differences between the spatial distribution of the $\mathrm{SiO}$ and the $\mathrm{HCO}^{+}$(Figure 2). The $\mathrm{SiO}$ emission in the $\mathrm{M}-3.8+0.9$ cloud has narrow profiles $\left(20 \mathrm{~km} \mathrm{~s}^{-1}\right)$ in comparison with the $\mathrm{HCO}^{+}$profiles $\left(50 \mathrm{kms}^{-1}\right)$, thus, shocked gas is dynamically more confined than the $\mathrm{HCO}^{+}$emission. In Figure 3 we show the integrated intensity ratio of the $\mathrm{SiO}$ to $\mathrm{HCO}^{+}$in velocity ranges of $10 \mathrm{~km} \mathrm{~s}^{-1}$. There are differences up to a factor 25 between the region where the $\mathrm{HCO}^{+}$and the $\mathrm{SiO}$ dominates (Riquelme et al., in prep).

\section{References}

Binney, J., Gerhard, O. E., Stark, A. A., Bally, J., \& Uchida, K. I. 1991, MNRAS 252, 210

Fukui, Y., Yamamoto, H., \& Fujishita, M., et al. 2006, Science 314, 106

Martín-Pintado, J., Bachiller, R., \& Fuente, A. 1992, A\&A 315

Martín-Pintado, J., de Vicente, P., Fuente, A., \& Planesas, P. 1997, ApJ Lett. 482, L45

Riquelme, D., Amo-Baladrón, M. A., \& Martín-Pintado, J., et al. 2013, A\&SA 549, A36

Riquelme, D., Amo-Baladrón, M. A., \& Martín-Pintado, J., et al. 2010, A\&A A 523, A51

Riquelme, D., Bronfman, L., Mauersberger, R., May, J., \& Wilson, T. L. 2010, A 8 A 523, A45 